\title{
Los mediadores literarios de los futuros maestros. Exploración de relatos de vida literaria'
}

\author{
The Literary Mediators of Future Teachers. \\ Exploring Stories of Literary Life
}

\author{
ELISABET CONTRERAS BARCELÓ \\ Universidad de Barcelona \\ España \\ econtreras@ub.edu
}

(Recibido: 29-O6-202O; aceptado: $2 \mathrm{I}-\mathrm{IO}-2 \mathrm{O} 2 \mathrm{O}$ )

Resumen. El presente trabajo forma parte de las investigaciones en el campo de la didáctica de la literatura sobre el perfil lector y el sistema de creencias del profesorado en formación inicial. Su objetivo es ahondar en la biografía literaria de los futuros enseñantes, prestando especial atención a quiénes fueron sus mediadores literarios, puesto que, posiblemente, acaben ejerciendo como modelos de mediación en su futura tarea como docentes. Para llevar a cabo dicha investigación se utiliza un Author Recognition Test, para establecer el grado de familiaridad de los estudiantes con la literatura, y el relato de vida literaria de los 35 estudiantes del doble grado de Educación Infantil y Primaria de la Universidad de Barcelona que configuran la muestra. Los resultados, que se construyen a partir de una aproximación cualitativa a los relatos de vida literarios y también de los datos cuantitativos del Author Recognition Test, muestran una relación con matices entre el grado de familiaridad de los estudiantes con la literatura y el tipo de mediador literario y las experiencias literarias vividos hasta el momento.

Palabras clave: Educación literaria; formación de profesores; mediación literaria.
Abstract. This work is part of the research in the field of teaching literature and the profile of the reader and the belief system of teachers in initial training. Its objective is to delve into the literary biography of future teachers, paying special attention to who their literary mediators were, since, possibly, they end up acting as mediation models in their future tasks as teachers. To carry out this research we use an Author Recognition Test to establish the degree of familiarity of students with literature, and the literary life story of the 35 students of the dual degree in Infant and Primary Education at the University of Barcelona, that configure the sample. The results, which are constructed from a qualitative approach to literary life stories and the quantitative data from the Author Recognition Test, show a nuanced relationship between the degree of familiarity of students with literature and the type of literary mediator and literary experiences lived so far.

Keywords: Literary education; teacher training; literary mediation.

\footnotetext{
${ }^{\text {I }}$ Para citar este artículo: Contreras Barceló, Elisabet (202I). Los mediadores literarios de los futuros maestros. Exploración de relatos de vida literaria. Alabe 24 . [www.revistaalabe.com]

DOI: I0.I5645/Alabe2O2I.24.5
} 


\section{Introducción}

La didáctica de la literatura como área de conocimiento ha evolucionado mucho a lo largo de las últimas décadas, así como sus líneas de investigación (Munita y Margallo, 20I9). Los vaivenes que ha sufrido desde los años 6o muestran la complejidad de una disciplina cuyo paradigma de enseñanza- aprendizaje ha cambiado notablemente. Ante este cambio, la formación de lectores literarios competentes se ha convertido en el objetivo principal de las clases de literatura. Para conseguirlo, la educación literaria debe centrarse en ayudar a los estudiantes en su devenir lector, aportándoles los conocimientos y habilidades suficientes para producir, comprender e interpretar un texto literario, así como la capacidad para establecer relaciones hipertextuales y elaborar un juicio argumentado sobre lo leído; sin olvidar la adquisición de unos hábitos de lectura, una actitud positiva hacia el hecho literario y la participación en el circuito social del libro (Mendoza, 2002; Munita, 2OI4). Se trata pues, de un objetivo ambicioso, cuya consecución requiere tiempo, esfuerzo y acompañamiento.

Esta educación literaria, tan necesaria en la formación del ciudadano moderno para fomentar el pensamiento crítico y entender mejor el mundo que lo rodea, trasciende el período de escolarización de los niños y niñas: se inicia en la cuna y se desarrolla a lo largo de la vida. A pesar de ello, será de vital importancia que durante su etapa de lector en formación, se nutra de obras de calidad, adecuadas al momento del aprendizaje lecto-literario, así como de experiencias estimulantes y, por supuesto, de personas que lo acompañen a lo largo del camino para facilitar los aprendizajes y transmitir el placer por la lectura. Y entre estas personas se encuentran, por supuesto, los docentes. Por lo tanto, hablar de mediación en educación literaria no es una cuestión menor.

La mayor responsabilidad a la hora de mediar entre este entramado de conocimientos, habilidades y actitudes, y los alumnos, recae en los docentes, cuyo objetivo debe ser crear lectores literarios competentes, con la ayuda de las familias y demás instituciones. Por esta razón, no es de extrañar que en los últimos años las investigaciones en didáctica de la literatura hayan puesto un foco de atención en los maestros en formación inicial, que son los futuros mediadores de la educación literaria: sus perfiles lectores, sus conocimientos, habilidades y actitudes. Los preocupantes resultados de dichas investigaciones han puesto en entredicho si su propia educación literaria es suficiente para convertirse en unos verdaderos mediadores de lectura literaria de las futuras generaciones (Colomer y Munita, 2OI3; Dueñas, Tabernero, Calvo y Consejo, 2OI4; Contreras y Prats, 20I5).

El presente artículo debe incluirse en esta línea de investigación dentro del ámbito de la didáctica de la literatura, y forma parte de los diversos estudios llevados a cabo por el grupo de investigación POCIÓ de la Universidad de Barcelona (2OI7 SGR I668) y del proyecto "Poéticas liminales en el mundo contemporáneo: creación, formación y compromiso social” (I+D) (PID20I9-IO4628RB-Ioo). 


\section{La mediación escolar de lectura literaria}

El cambio de paradigma en la enseñanza-aprendizaje de la literatura supuso un replanteamiento de la figura del docente, de modo que dejó de ser concebida solo como un profesor transmisor de conocimientos para empezar a adquirir también un rol de mediador. Este concepto tiene sus raíces en la teoría vygotskiana de las zonas de desarrollo del niño y no es un simple cambio semántico, sino un verdadero cambio conceptual: el profesor solo transmite conocimientos, mientras que el mediador también ayudará en el progreso de aprendizaje del alumno, creando el contexto propicio, con instrumentos y estrategias adecuadas (Mendoza, 2004; Colomer, 2006). Por eso, el docente que ejerce de mediador actúa con intencionalidad, trascendencia y significado, convirtiéndose así en un verdadero puente entre el alumno y su propio aprendizaje (Feuerstein y Feuerstein, I99I).

El aprendizaje de la lectura es complejo y requiere la instrucción de un lector experto. El aprendiz necesita a alguien que, en palabras de Vygotski (2009), le acompañe hacia la zona de desarrollo próximo, para que más tarde pueda hacerlo solo. En esta línea, Munita y Manresa (2OI2) destacan la importancia de la mediación literaria para la adquisición de la competencia lectora y literaria:

El acercamiento del niño a los libros y su entrada al campo literario, así como la progresiva apropiación de sus particularidades discursivas, necesitan de unos adultos que mediaticen el objeto de aprendizaje (el discurso literario), dotándolo de sentido en el contexto de la actividad de niños y niñas. (Munita y Manresa, 2OI2: I2O)

Este proceso se da en dos contextos: el familiar y el social (escuela, bibliotecas y demás instituciones y entidades), y en cada entorno habrá agentes que ejercerán de mediadores, en tanto que contribuirán a la aproximación entre los lectores noveles y los textos escritos (Manresa, 20I3). A pesar de que la familia, las bibliotecas, librerías y demás entidades facilitan el encuentro entre los niños y los libros y los animan a su lectura, es la escuela quien debe desempeñar la tarea de mayor envergadura: acompañar a los estudiantes en su formación como lectores competentes.

En 2002, el profesor Mendoza reivindicaba el cambio de paradigma pedagógico en las clases de literatura (sobre todo en secundaria), que debían dejar de basarse únicamente en la transmisión enciclopédica de autores y obras -metodología tradicional-, si se pretendía que los estudiantes adquirieran una competencia literaria (y lectora) -educación literaria- y aprendieran a apreciar la literatura. Asimismo, esbozaba las funciones que debía asumir el docente en el nuevo paradigma de educación literaria: seleccionar, dinamizar e interpretar textos, así como animar a la lectura y mostrarse como un modelo lector ante sus estudiantes. En otras palabras, el docente debía convertirse en facilitador de los conocimientos, habilidades y actitudes: un mediador escolar de lectura literaria. 
Para ello, no se trata de restringir la transmisión de conocimientos, sino de añadir otras funciones para poder abarcar la complejidad y diversidad de objetivos que implica el nuevo paradigma (Naji, Subramaniam y White, 20I9; Almeida, Bavendiek y Biasini, 2020). Así constata Munita (2OI4) cómo debe ser el rol de este mediador escolar de lectura literaria:

El docente multiplica sus intervenciones, que van desde la planificación de espacios que permitan la confrontación de interpretaciones, la reflexión metacognitiva y la sistematización de saberes, hasta otros que garanticen el acceso a una amplia diversidad de textos y que favorezcan la participación en circuitos reales de circulación y mediación de la literatura. (Munita, 20I4: I58)

\section{Los mediadores escolares de lectura literaria en formación inicial}

A partir de la multiplicidad de frentes que debe atender en materia literaria para facilitar y guiar a los estudiantes en su educación literaria, el docente necesitará poner en práctica saberes disciplinares, estrategias didácticas para dinamizar la lectura en el aula, criterios para seleccionar las obras adecuadas y ejercer de modelo. Sin embargo, más allá de los saberes en materia literaria y en didáctica, la identidad profesional del mediador se verá condicionada también por otros elementos como la reflexión sobre sus propias prácticas de lectura y sus creencias sobre la literatura y su enseñanza a partir de sus propias experiencias y modelos docentes asimilados (Munita 2OI4).

En este punto se debe abordar la noción del "sujeto lector didáctico" (Delbrayelle y Duszynski, 2007; Munita, 20I8) que aborda la compleja relación entre la lectura personal del maestro y sus prácticas docentes. Se trata de un supuesto ampliamente aceptado por la comunidad científica, basado en que aquellos maestros que tienen éxito en la formación de lectores competentes también disfrutan de sus lecturas personales y comparten su entusiasmo con los alumnos. La formación de lectores literarios tiene una dimensión emocional que va más allá del ser un ejemplo de lector que comparte con sus estudiantes las estrategias y conocimientos, se trata de contagiar ese placer y compartir su participación en el circuito social del libro (Applegate, Applegate, Mercantini, McGeehan, Cobb, DeBoy, Modla, Lewinski, 2OI4; Sanjuán 20I6). De este modo,

La noción de "sujeto lector didáctico" recoge la interacción entre ambas dimensiones, la personal y la profesional, y ayuda a focalizar en la compleja dialéctica que caracteriza la puesta en juego de las posiciones de actor y mediador en las prácticas de lectura, particularmente en el ámbito literario. (Munita, 2OI4: 62) 
Más allá de la relación entre el docente como lector en su espacio personal y sus propias prácticas profesionales, hay otro elemento que contribuye en la construcción de la identidad profesional del mediador: sus experiencias personales pre-profesionales. Según recogen Trotman y Kerr (200I), las propias vivencias como estudiantes suponen una resistencia durante su posterior formación como maestros. Aquello que experimentaron es aceptado a menudo como un status quo y se convierte en una creencia difícil de modificar. Así, las creencias sobre la lectura se han ido configurando a través de sus propias experiencias y actitudes a lo largo de su vida, del mismo modo que las prácticas docentes vividas son tomadas como modelos. De este modo, la propia biografía personal se convierte en una gran influencia en la construcción de la identidad del docente, que debe tenerse en cuenta y abordarse durante su formación universitaria (Díaz-Plaja y Prats 20I3; Granados y Puig 2OI5; Berríos Barra, 20I8). A partir de la problematización de las propias creencias, la reflexión y la experimentación de prácticas innovadoras y significativas pueden revertir visiones negativas de la lectura literaria.

A partir de estas consideraciones y teniendo presente los diversos estudios que se aproximan a la educación literaria de los futuros maestros, que muestran unos resultados desoladores (Larrañaga y Yubero 2005; Colomer y Munita, 20I3; Dueñas (et al.) 20I4; Contreras y Prats 2OI5), el presente artículo explora los relatos de vida literaria de un grupo de estudiantes de Educación Infantil y Primaria. El objetivo principal de la investigación será ahondar en la educación recibida en este ámbito a lo largo de su vida, prestando especial atención a quiénes fueron sus mediadores, así como a las experiencias positivas o negativas que recuerdan y su actitud hacia la literatura. A partir de aquí, nos preguntaremos sobre las relaciones entre el grado de familiaridad que tiene el estudiante con la literatura y el tipo de mediador que tuvo, para establecer una relación entre el tipo de experiencia que relata, su actitud hacia la lectura y los mediadores.

\section{Metodología}

Como parte de una investigación más amplia, se llevó a cabo un estudio con el objetivo de aproximarnos a la educación literaria de un grupo de 35 estudiantes del doble grado de Educación Infantil y Primaria de la Universidad de Barcelona, para conocer quiénes ejercieron de mediadores literarios durante sus etapas formativas anteriores. Asimismo, se pretende detectar si las experiencias satisfactorias en relación con la literatura han estado ligadas a estos mediadores y también observar la posibilidad de establecer una relación entre el grado de familiaridad de cada estudiante con el tipo de mediador que han tenido y su actitud hacia la lectura literaria.

Se trata de un estudio que sigue un diseño de triangulación concurrente en el que se aplicaron dos instrumentos para la recogida de datos: un Author Recognition Test (Stanovich y West, 1989), para establecer de manera cuantitativa el grado de familiaridad de los estudiantes con la literatura; y un relato de vida, entendido como "la narración 
retrospectiva por el propio protagonista" (Navarro Asensio 2OI7: 150), con lo cual los datos obtenidos en este caso proporcionarían datos que profundizarían y matizarían los obtenidos en el estudio cuantitativo.

De este modo, la triangulación de los datos ayudaría a obtener una visión más amplia de la realidad estudiada (Sandín, 2003).

\section{Participantes y desarrollo de la investigación}

El estudio se realizó en el marco de la asignatura obligatoria de $3^{\circ}$ curso, Didáctica de la Literatura Infantil y Juvenil, del doble grado de Educación Infantil y Primaria de la Universidad de Barcelona, durante el curso 20I8-20I9. De un total de 44 alumnos matriculados en el curso, participaron 35 (34 mujeres y I hombre), que dieron su consentimiento informado para formar parte de esta muestra parcial pero significativa del grupo (el 79,54\% del total de estudiantes).

Durante las primeras sesiones del semestre se interpeló a los estudiantes acerca de su relación con la literatura, en un debate abierto para introducir el tema. A continuación se aplicó a la muestra seleccionada un Author Recognition Test (ART a partir de ahora) para determinar el grado de familiaridad de los estudiantes con la literatura. A pesar de que sus autores, Stanovich y West (I989) idearon el instrumento con una finalidad psicolingüística, ha sido posteriormente validado para medir el grado de relación entre una persona y la literatura (Mar, Oatley, Hirsh, dela Paz y Peterson, 2oo6; Rain y Mar, 2OI4). El ART consiste en una lista de 80 nombres de escritores, de los cuales solamente $4 \mathrm{O}$ son reales y los alumnos tienen que identificarlos. En nuestro caso se adaptó la versión del ART al contexto de los estudiantes y se escogieron autores de todos los géneros: clásicos de la literatura catalana, española y universal, de literatura infantil y juvenil, de bestsellers y mediáticos -nacionales e internacionales que han sido traducidos al español o catalán-. Dicha selección fue avalada por un grupo de 4 expertos en literatura y su didáctica del grupo de investigación POCIÓ de la Universidad de Barcelona. La lista de autores se completó con 40 nombres falsos, obteniendo así, un total de 8o. Se facilitó el documento a los 35 estudiantes y se les explicó en qué consistía. Asimismo, se les informó que con cada acierto obtenían un punto positivo y con cada error perdían un punto; de modo que los resultados del test podían oscilar entre -40 y 40 . Estos realizaron el test en el aula, sin acceso a dispositivos conectados a la red para evitar que se alterara el resultado.

Al finalizar el test, se planteó a los alumnos la redacción de un relato de vida literaria, bajo el título "Autobiografía literaria” y con una única instrucción: “Cuéntanos cuál ha sido tu recorrido literario". A partir de esta propuesta, poco concreta a propósito para no condicionar la respuesta, debían elaborar una narración retrospectiva en primera persona, a través de la cual irían desgranando la educación literaria recibida hasta el momento: primeros contactos con la literatura, libros amados y odiados, actividades y personas que interfirieron para bien o para mal, así como hábitos, aprendizajes y emociones. El relato de vida se ha convertido en un instrumento clave en las investigaciones, especialmente en el ámbito de la educación (Palou y Fons, 2OIO), por su capacidad de 
mostrar los sucesos que conforman la historia personal de cada cual, con el significado y el sentido que este le otorga. Asimismo, son una estrategia de reflexión muy interesante para la formación docente, porque confronta a los estudiantes con sus propias creencias sobre la educación literaria y los ayudan a tomar consciencia de sus necesidades como futuros maestros (Munita 20I3).

Así pues, los datos de la investigación se construyeron a partir de los resultados cuantitativos del ART y del análisis cualitativo de las reflexiones del relato de vida. Por lo que se refiere al ART, se corrigieron los test y, a partir de los resultados obtenidos, se crearon 3 categorías de manera inductiva (Creswell, 20I2) para establecer el grado de familiaridad (tabla I):

Tabla I. Relación de categorías de análisis del ART

\begin{tabular}{|c|c|}
\hline Puntuación & Grado de familiaridad \\
\hline Entre 20 y 25 & Medio \\
\hline Entre 19 y 10 & Bajo \\
\hline Entre 9 y 0 & Muy bajo \\
\hline
\end{tabular}

Fuente: Elaboración propia.

En cuanto a los datos procedentes de los relatos de vida literaria, fueron tratados de manera cualitativa a través del software NVivo (QSR International Pty Ltd, 2OI6). Con dicho programa se han podido procesar los datos y crear tres grandes categorías de análisis también de manera inductiva (Creswell 2OI2): mediadores, experiencias y actitudes, que a su vez se dividen en subcategorías. 
Tabla 2. Relación de categorías de análisis del relato de vida literaria.

\begin{tabular}{|c|c|}
\hline Categoría & Descripción de la categoría \\
\hline Mediadores & $\begin{array}{l}\text { Personas que han intervenido en su educación literaria, ya } \\
\text { sea en un contexto escolar, familiar o social (bibliotecas, } \\
\text { librerías, etc.). }\end{array}$ \\
\hline Mediadores positivos & $\begin{array}{l}\text { Personas que, según el participante, han contribuido a forta- } \\
\text { lecer el vínculo entre el participante y la literatura. }\end{array}$ \\
\hline Mediadores negativos & $\begin{array}{l}\text { Personas que, según el participante, les ha alejado de la } \\
\text { lectura literaria. }\end{array}$ \\
\hline Experiencias & $\begin{array}{l}\text { Experiencias relacionadas con la literatura: aprendizajes, } \\
\text { lecturas, actividades... }\end{array}$ \\
\hline Experiencias positivas & $\begin{array}{l}\text { Experiencias relacionadas con la literatura de las que se } \\
\text { guarda un recuerdo positivo. }\end{array}$ \\
\hline Experiencias negativas & $\begin{array}{l}\text { Experiencias relacionadas con la literatura de las que se } \\
\text { guarda un recuerdo negativo. }\end{array}$ \\
\hline Actitudes & $\begin{array}{l}\text { Autoimagen de lectores, qué tipo de lector se consideran. } \\
\text { Hábitos y gustos. }\end{array}$ \\
\hline Lector habitual & El sujeto se considera a sí mismo lector habitual. \\
\hline Lector ocasional & $\begin{array}{l}\text { El sujeto se considera a sí mismo lector ocasional o irregu- } \\
\text { lar. }\end{array}$ \\
\hline No lector & El sujeto se considera a sí mismo no lector. \\
\hline Falso lector & $\begin{array}{l}\text { El sujeto muestra contradicción entre un discurso lleno de } \\
\text { tópicos socialmente aceptables sobre la lectura y falta de } \\
\text { hábitos de lectura. }\end{array}$ \\
\hline
\end{tabular}

Fuente: Elaboración propia.

\section{Resultados}

Los resultados de la investigación se construyeron a partir de los datos cuantitativos del ART y del análisis cualitativo de los relatos de vida literaria, a través del programa NVivo (QSR International Pty Ltd, 20I6) y de la triangulación de ambos tipos de datos para atender a los objetivos planteados. 


\section{Los mediadores de lectura literaria}

$\mathrm{Al}$ aproximarnos a los relatos de vida literaria de los participantes, se puede observar cómo afloran aquellas personas que los han acompañado en su viaje. Hasta en un 77,I4\% casos los participantes mencionan al menos a una persona que ha intervenido en su educación literaria, frente al 22,85\% de los participantes que no nombra a nadie. Si nos fijamos en las personas que se mencionan en los relatos de vida, se puede observar, tal y como muestra el gráfico siguiente, cómo la mayoría pertenecen al ámbito familiar $(64,6 \%)$, seguidas de los docentes $(29,2 \%)$ y los amigos $(6,3 \%)$.

Gráfico I: Mediadores de lectura literaria.

\section{Mediadores de lectura literaria}

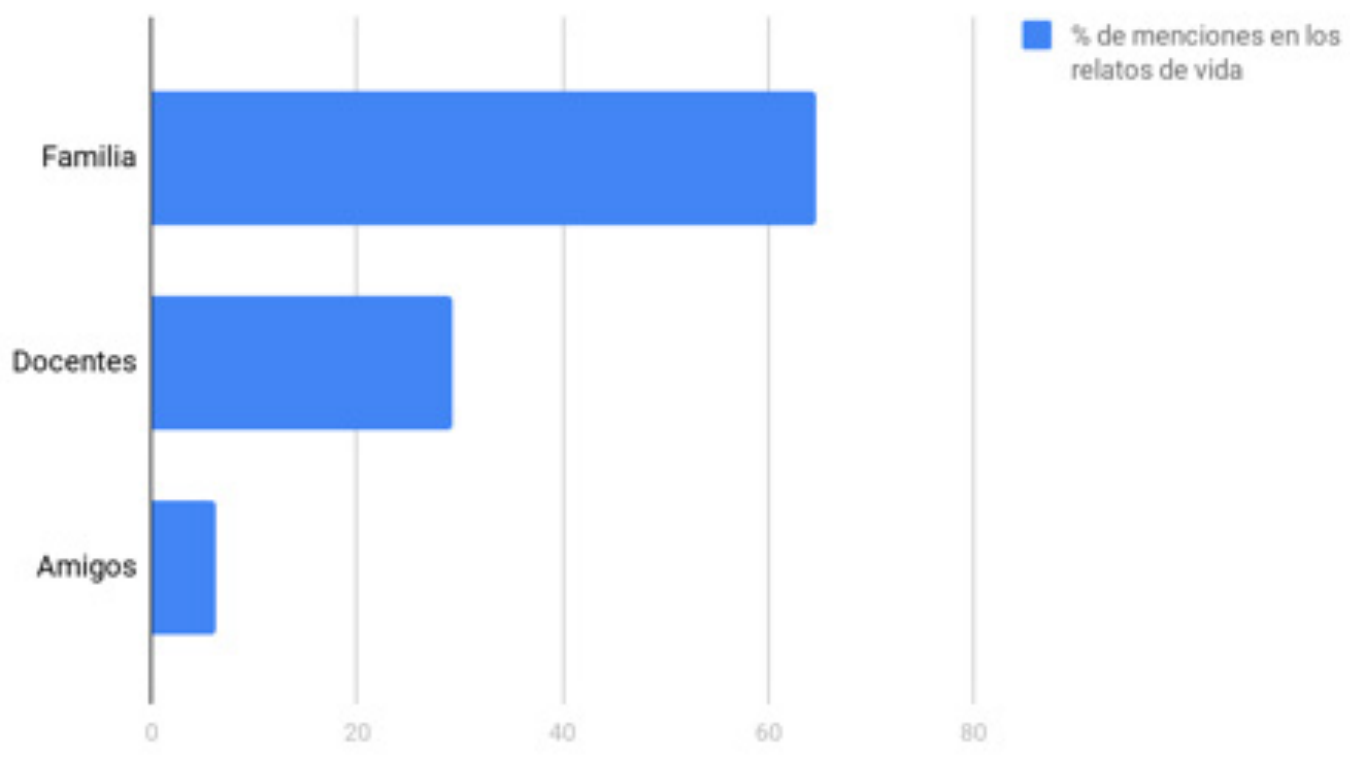

Dentro del ámbito familiar, la madre y el padre juegan un papel muy importante en la educación literaria de estos estudiantes (especialmente la madre), seguidos de abuelos, hermanos y tíos. Estos son considerados mediadores positivos, pues los participantes guardan un buen recuerdo de todos ellos.

En el 29,I6\% de los casos en que mencionan algún mediador literario, los participantes destacan el papel de los docentes. En estos casos, aparecen mediadores que han contribuido a fortalecer su vínculo con la literatura -mediadores positivos-, pero también encontramos docentes cuya labor consideran que les ha alejado -mediadores negativos-. Tal y como puede observarse en el gráfico 2, de entre aquellos casos que se menciona a los docentes, no hay ninguna que sea de educación infantil, el $35,7 \%$ nombra a maestros de educación primaria, de quienes algunas veces no guardan un buen recuerdo, y en la gran mayoría (el 64,8\% de los casos) aparecen docentes de secundaria y bachillerato, de quienes guardan un buen recuerdo. 
Gráfico 2: Los docentes como mediadores literarios.

\section{Los docentes como mediadores literarios}

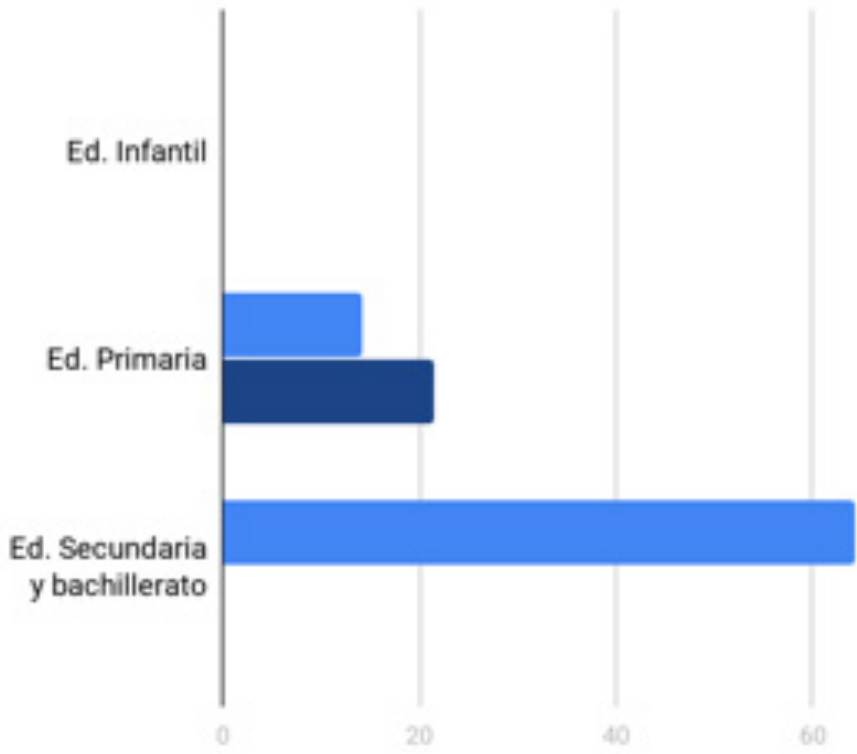

S Mediadores positivos

\$ Mediadores negativos

Véase algunos testimonios sobre los docentes como mediadores, cuyas vivencias se repiten de manera parecida en varios participantes:

"Al llegar a bachillerato y hacer la asignatura de literatura catalana volví a sentir aquel amor por la literatura, ya que la profesora transmitía su entusiasmo y me lo hacía vivir. (...) puedo decir que gracias a la asignatura pude revivir aquello que sentía de pequeña cuando leía." -Estudiante id. I7.

"Pero cuando empecé el colegio, leer se convirtió en una cosa aburrida, obligada por los maestros, títulos de libros que no me interesaban.” -Estudiante id. 34

Finalmente, el 6,3\% de los participantes que mencionaron algún mediador destacaban el papel ejercido por algún amigo. En todos los casos estos amigos son de su misma edad y guardan de ellos un buen recuerdo, sobre todo de sus recomendaciones de lectura.

Relación entre el grado de familiaridad con la literatura, el mediador literario y la actitud frente a la lectura

El análisis de los resultados del ART mostraron que los participantes, en general, presentan un grado de familiaridad bajo con la literatura: solamente el I $4,2 \%$ superó el test con una puntuación de entre 20 y 25 sobre 4O, mientras que el 74,2\% obtuvo una puntuación de entre i9 y Io puntos y el II,4\% ni siquiera llegó a los Io puntos.

Si se triangulan los resultados cuantitativos del ART con los resultados cualitativos sobre los mediadores literarios en los relatos de vida, se puede profundizar en la 
educación literaria que han recibido los participantes. En el siguiente gráfico (Gráfico 3), puede observarse quiénes aparecen como mediadores en los relatos de vida de cada estudiante, en función de la puntuación que obtienen en el ART.

Gráfico 3: Mediadores según la puntuación en el ART.

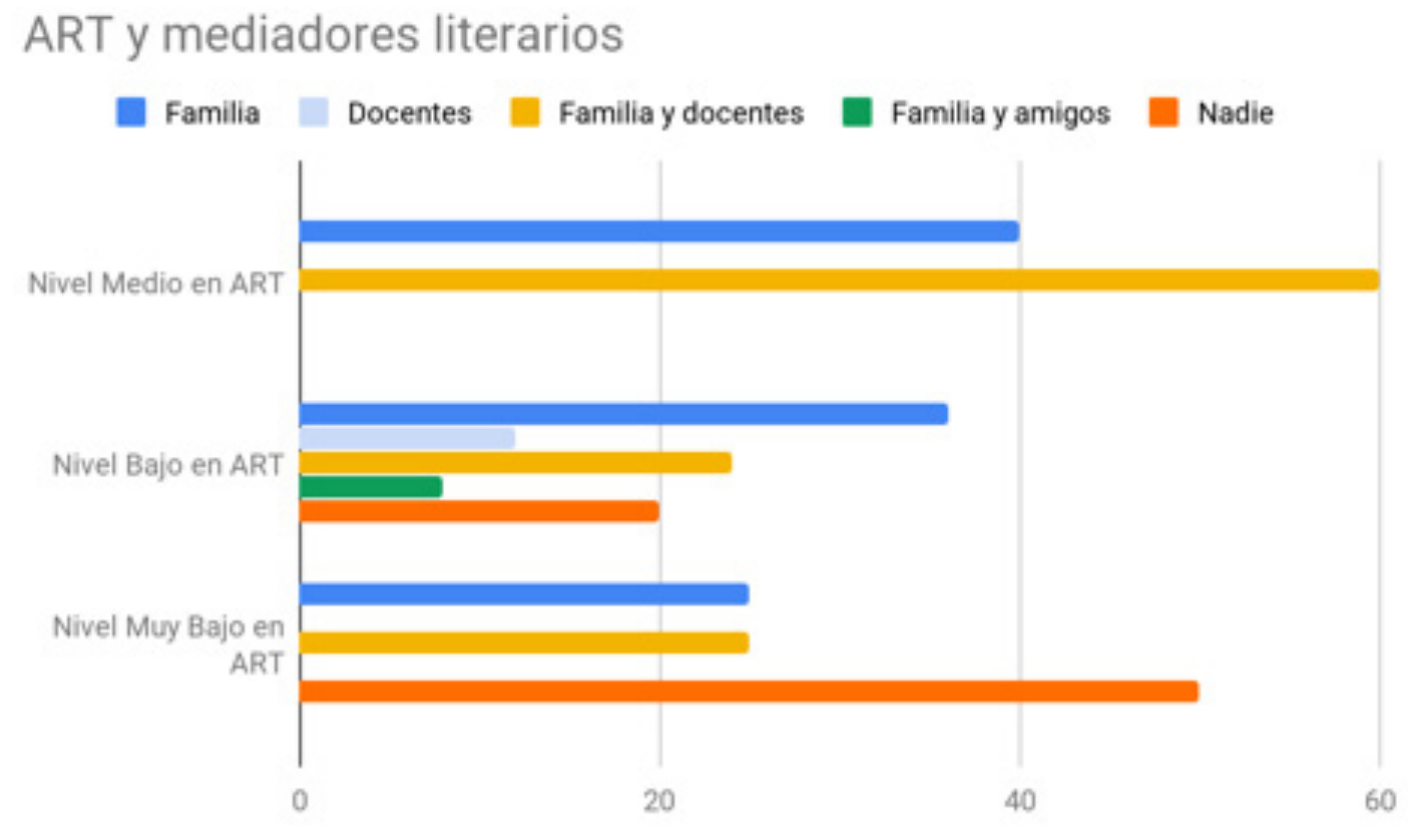

Si se atiende al tipo de mediador literario que mencionan los estudiantes que obtuvieron entre 20 y 25 puntos, todos nombran al menos a un mediador en sus relatos de vida. El 6o\% de los casos mencionan a alguien de la familia y a algún docente y el $40 \%$ solamente a un familiar. En este grupo de participantes, que representa el I $4,2 \%$ del total de la muestra, el $60 \%$ se consideran lectores habituales y el $40 \%$ se definen como lectores ocasionales. Véase el testimonio de un par de estudiantes cuyas vivencias experiencias aparecen de manera parecida en varios participantes:

"Guardo un buen recuerdo del libro, ya que la profesora de sexto decidió que los viernes por la tarde, a la hora de tutoría, en lugar de avanzar en los deberes o jugar, leeríamos todos juntos." -Estudiante id. 8

"Tengo la sensación de haber leído pocos libros por voluntad propia debido a que en el instituto nos hacían leer mucho." -Estudiante id.4I.

Los estudiantes con un grado de familiaridad bajo forman un grupo amplio y heterogéneo (74,2\% de la muestra). El I5,38\% de estos no nombra a nadie, ni familiares ni docentes ni amigos, mientras que el 84,62\%, sí. De entre los mediadores más mencionados en sus relatos, se encuentran también sus familias (6I,II\%), y cobran especial importancia las madres. Los docentes también se mencionan a menudo $(30,55 \%)$ y la mayoría 
aparecen ligados a recuerdos positivos; sin embargo, en algunas ocasiones los maestros de primaria se vinculan a recuerdos negativos relacionados con el aprendizaje de la lectura (2,75\%). También aparecen los amigos que representan un 5,55\% de los mediadores. Véase un ejemplo paradigmático en este grupo:

"Mi madre siempre me ha dicho que de pequeña era una entusiasta de la literatura, una actividad que he ido perdiendo con los años (...) Los años en que verdaderamente he disfrutado de la literatura fueron los de bachillerato (...) tuve una profesora que nos enseñó a ir más allá, a leer entre líneas e interpretar historias.” - Estudiante id. 37.

Si se atiende a cuál es su actitud frente a la lectura, el 46,I5\% de los participantes de este grupo se consideran no lectores. De entre los que dicen ser lectores, el 7,69\% se consideran lectores ocasionales y el I9,23\% se consideran lectores habituales. Un $26,92 \%$ de estos estudiantes elogian los beneficios de la lectura, pero se muestran muy exigentes a la hora de coger un libro.

En el tercer y último grupo de estudiantes, cuyo ART no superó los 9 puntos de los 40 posibles, se encuentra el II,4\% de la muestra. El $50 \%$ de estos estudiantes no mencionó a ningún mediador literario, la otra mitad del grupo mencionó en un 25\% a la madre y en otro $25 \%$ a algún docente para culparlo de su poco gusto por la lectura. Véase un ejemplo:

“De muy pequeña mis padres y mis abuelos me leían cuentos y me contaban historias

y yo estaba muy atenta. Pero cuando empecé el colegio, leer se convirtió en una cosa aburrida, obligada por los maestros.” -Estudiante id.34.

Si nos fijamos en qué tipo de lector se consideran, se observa que en el $75 \%$ de estos casos se consideran no lectores, a través de un relato en el que se justifican, y muestran una voluntad más o menos explícita para intentar mejorar sus hábitos de lectura.

\section{Relación entre las experiencias vividas, los mediadores literarios y el grado de familiaridad con la literatura}

Al observar qué tipo de actividades relacionadas con la literatura cuentan los estudiantes en los relatos de vida, se han podido clasificar entre experiencias positivas y negativas, en función de si asocian la vivencia a un buen recuerdo o a un mal recuerdo.

Es un hecho que la gran mayoría de los recuerdos que reportan son de experiencias positivas $(88,05 \%)$. De estas, el 71,18\% están vinculadas al entorno familiar y el 28,81\% están relacionadas con su formación académica. Por lo que se refiere a las negativas, que solamente ascienden al Iı,95\%, el 75\% aparecen vinculadas al ámbito escolar y el $25 \%$ al ámbito del hogar.

Si nos detenemos en la relación entre el grado de familiaridad con la literatura y el tipo de experiencias que han reportado los participantes, tal y como muestra el gráfico 4 , 
el Ioo\% de los estudiantes que obtuvieron un nivel medio del ART, reportan experiencias positivas, de las cuales el $72,72 \%$ tuvieron lugar en el ámbito familiar y el $27,27 \%$ en el ámbito escolar. De entre aquellos que obtienen un nivel bajo, el 90,2\% recuerda también experiencias positivas, de estas el $69,56 \%$ vinculadas al entorno familiar y el $30,43 \%$ al escolar. El 9,8\% de estudiantes que reportaron una experiencia negativa siempre están relacionadas al contexto escolar. Finalmente, los participantes con un nivel muy bajo en el ART, el $40 \%$ relata experiencias positivas, siempre vinculadas a la familia, mientras que el 6o\% reporta experiencias negativas, de las cuales un 33,33\% tuvieron lugar en la escuela y un $66,66 \%$ en el entorno familiar.

Gráfico 4: Experiencias y mediadores literarios.

\section{Experiencias y mediadores literarios}

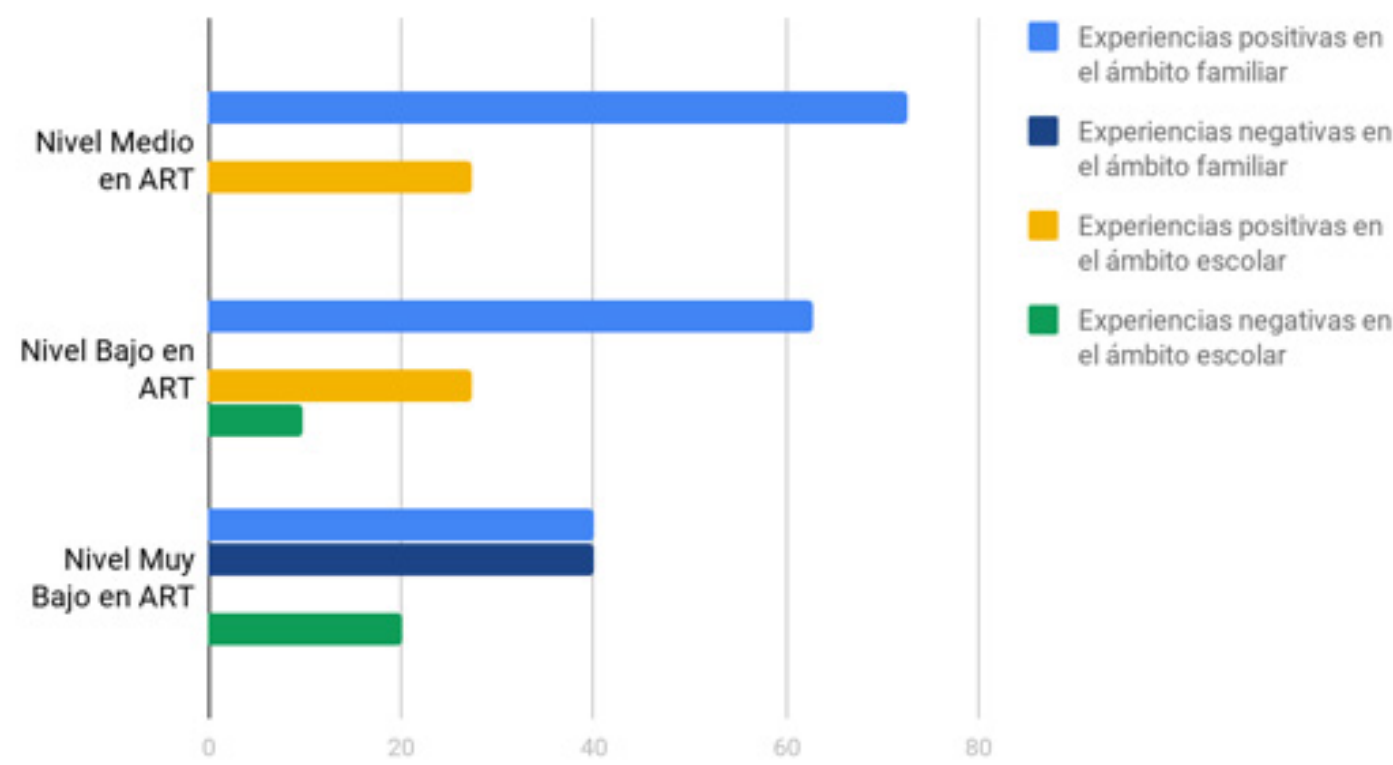

La mayoría de buenos recuerdos están relacionados con mediadores del entorno familiar y especialmente vinculados a la primera infancia. Véase algunos ejemplos que se van repitiendo en la mayoría de relatos de vida:

“Siempre agradeceré que mi madre me obligara a acompañarla a la librería." -Estudiante id. I

"Mi madre me acostumbró a dormirme y despertarme con un cuento." -Estudiante id.5

Tal y como se apuntaba, en relación a las experiencias en el contexto académico, la mayoría son positivas y muchas de estas experiencias se relacionan a sus estudios de bachillerato. Mientras que las experiencias pertenecientes a la etapa de primaria y secundaria obligatoria son de animación a la lectura, las que hacen referencia al bachillerato están relacionadas con la competencia interpretativa y la familiarización con el discurso y la tradición literaria. 
Si atendemos a las experiencias negativas que cuentan los estudiantes, estas corresponden en su totalidad a su paso por la primaria y la secundaria obligatoria y están relacionadas con el aprendizaje de la lectura y las lecturas obligatorias, incluso si los malos recuerdos tienen lugar en el ámbito familiar, estos remiten a deberes y tareas del colegio.

\section{Discusión}

A partir del análisis de los resultados obtenidos del relato de vida y del ART y de su triangulación se ha podido profundizar en la educación literaria de los participantes.

En primer lugar, se ha constatado hasta qué punto son importantes las personas que ejercen de mediadores en la educación literaria de uno, pues hasta en un 77,I4\% de los relatos de vida aparecen mediadores, y cómo estos ejercieron de puente entre los participantes y sus aprendizajes literarios (Feuerstein y Feuerstein I99I), tal y como exponen los estudios de Munita y Manresa (2OI2) y Munita (2OI4).

Los mediadores que aparecen en los relatos de vida provienen de los contextos en los que se desarrolla el aprendizaje de la lectura literaria: el familiar y el social (Manresa 20I3). Aunque el contexto familiar está ampliamente representado por distintos miembros $(64,5 \%)$, del contexto social solamente aparecen docentes $(29, \mathrm{I} 6 \%)$ y amigos $(6,25 \%)$, pero en ningún caso se mencionan libreros, bibliotecarios ni otras personas del circuito social del libro.

En segundo lugar, los resultados del ART mostraron que, en su gran mayoría, los participantes presentan un grado de familiaridad bajo con la literatura, y su actitud frente a la lectura y su autoimagen como lectores también está en sintonía con otros estudios sobre perfiles lectores y competencia literaria de futuros maestros (Colomer y Munita, 2OI3; Dueñas (et al.) 2OI4; Contreras y Prats 2OI5). Incluso se han podido detectar falsos lectores, quienes en sus relatos de vida han elaborado un discurso socialmente aceptable a base de tópicos sobre la lectura, pero cuyos hábitos no corresponden con dicho discurso (Larrañaga y Yubero, 2005).

Al triangular los datos del relato de vida con los resultados del ART, se ha podido establecer relaciones entre los mediadores de los participantes con su grado de familiaridad con la literatura, las experiencias evocadas y su actitud frente a la lectura, y ha permitido obtener así mayor conocimiento de la realidad estudiada.

En la muestra seleccionada, aquellos participantes que obtuvieron mejor puntuación en el ART y su autoimagen de lector es más positiva son aquellos que reportan más casos de doble mediación entre la familia y los docentes, aunque consideran la familia el pilar fundamental. Asimismo, sus experiencias y recuerdos en relación a la literatura son siempre positivos, tanto los relacionados con la escuela, como con la familia.

Mientras, en el grupo de nivel bajo en el ART, aparecen algunos casos en que no se menciona ningún mediador, y la conjunción entre familia y docentes desciende significativamente. Sin embargo, la familia sigue llevando el peso de la mediación literaria. La 
gran mayoría de sus recuerdos son positivos, tanto en el ámbito escolar como familiar, aunque algunos casos reportan experiencias negativas durante su aprendizaje de la lectura.

Sin embargo, en el grupo de estudiantes con un nivel muy bajo de familiaridad con la literatura y de autoimagen lectora, los casos en que no han vinculado ningún mediador a su educación literaria ascienden hasta la mitad y solo en un $25 \%$ se menciona a docentes. En esta misma línea, los recuerdos que relatan están ligados en su mayoría a experiencias negativas relacionadas con el aprendizaje de la lectura o las lecturas obligatorias.

A partir de los datos analizados de la muestra compuesta por los 35 participantes, se puede constatar que el mediador es la figura clave en la educación literaria (Munita 20I4), pues a medida que desciende el grado de familiaridad con la literatura ascienden los casos que no reportan ningún mediador. La familia se erige en un pilar fundamental en la mediación literaria, como facilitadores de encuentros entre los niños y los libros en todos los niveles del ART. Sin embargo, proporcionar libros y contar cuentos no parece suficiente para adquirir competencia en lectura literaria, pues tal y como muestran los datos, aquellos que obtuvieron una mayor puntuación en el ART son quienes tuvieron a su familia y sus docentes como mediadores, cada cual en su entorno. Según los relatos de vida literaria, parece que los mediadores escolares de los participantes no fueron tan significativos como la familia, y a pesar de ello cabe la posibilidad de que se hayan convertido en modelos de mediación (Trotman y Kerr, 20oI; Díaz-Plaja y Prats, 20I3). Asimismo, las experiencias que recuerdan están más vinculadas a la animación a la lectura y al hábito lector, que a la competencia lecto-literaria (Mendoza, 2004). A pesar de que en los cursos de didáctica de la literatura se insiste en que este tipo de prácticas no son suficientes para adquirir la competencia literaria (Mendoza 2002; Munita y Manresa 2OI2; Munita 20I4), puede que hayan sido tomadas como status quo.

Si tenemos en cuenta que la identidad del mediador de lectura literaria está condicionada por las experiencias pre-profesionales, que pueden convertirse en creencias con resistencia al cambio (Trotman \& Kerr 2OOI), ya sean sus experiencias con la lectura o los docentes que ejercieron como modelos en mediación literaria (Díaz-Plaja \& Prats 20I3), sus creencias actuales sobre la lectura o sus propias prácticas como lector (Munita 2OI4; Sanjuán 2OI6; Applegate (et al.) 2OI4), todo nos hace pensar que las experiencias de mediación literaria escolar de los participantes no han resultado tan relevantes como deberían haber sido. Finalmente, es necesario señalar que cabría ampliar la muestra para constatar hasta qué punto se puede generalizar esta situación. 


\section{Conclusiones}

La presente investigación ha permitido profundizar en la realidad de un contexto determinado. Con una muestra de 35 participantes, sus resultados no pueden extrapolarse al total de estudiantes de Educación ni mucho menos, pero nos ha permitido aproximarnos a la educación literaria de estos alumnos, explorar las propias experiencias que moldearon su identidad de futuros mediadores de lectura literaria, a través de sus experiencias con la lectura y los mediadores que ejercieron como modelos, tal y como ellos lo vivieron, teniendo presente el grado de familiaridad con la literatura de cada cual.

Este estudio nos ha permitido constatar la importancia de los mediadores en los aprendizajes literarios y la relación que se establece entre estos mediadores y el grado de familiaridad con la literatura. Se ha podido observar de qué manera la familia siempre es el primer mediador que mencionan. Sin embargo, en los casos de mayor éxito, además de la familia, también hay docentes que los acompañan. Asimismo, cuanto más bajo es el grado de familiaridad del estudiante con la literatura, menos mediadores han intervenido y menos experiencias positivas recuerdan con la lectura.

Si estos elementos condicionan la identidad del futuro mediador literario, como se ha visto en estudios previos, cabe la posibilidad de encontrarnos en una situación circular: los estudiantes han tenido mediadores escolares de literatura poco significativos para ellos, pero posiblemente se hayan convertido en sus modelos de todos modos, y cuando ejerzan de mediadores sean también poco significativos para sus alumnos. De esta manera, los lectores literarios competentes serán aquellos cuya familia les haya podido familiarizar con el discurso y la tradición literaria.

En conclusión, ante tal situación se debe actuar para romper esta dinámica; los docentes deben ser el pilar fundamental de la educación literaria de los lectores en formación para garantizar la igualdad de oportunidades entre todos los alumnos. Por supuesto que la familia juega un papel muy importante, y así debe seguir siendo, pero la mediación escolar de lectura literaria debe ser más significativa y trascendente, empezando por dotar a los docentes en formación inicial de modelos de mediación capaces de ayudar a sus futuros alumnos a construir el significado de los textos, ampliar su intertexto lector, guiarlos hacia una lectura literaria expertay, en definitiva, ayudarles en su adquisición de la competencia literaria. 


\section{Bibliografía}

- Almeida, A.B; Bavendiek, U.; Biasini, R. (Ed.) (2020). Literature in Language Learning: new approaches. Francia: Research-publishing.net.

https://doi.org/IO.I47O5/rpnet.2020.43.9782490057696

-Applegate, A.J.; Applegate, M.; Mercantini, M.A.; McGeehan, C.M.; Cobb, M.J.; DeBoy, J.R.; Modla, V.B. y Lewinski, K.E. (20I4). The Peter Effect Revisited: Reading Habits and Attitudes of College Students. Literacy Research and Instruction, 53, I88-204. https://doi.org/IO.IO80/I938807I.20I4.898719

- Berrios Barra, L. (2018). Una experiencia didáctica a partir del hipertexto digital y la multimodalidad con profesores de lengua y literatura: aproximación de los clásicos a los adolescentes. (Tesis doctoral). Obtenido desde Dipòsit Digital UB en 2O2O:

http://diposit.ub.edu/dspace/handle/2445/ı28487

- Colomer, T. (2006). Andar entre libros: la lectura literaria en la escuela. México: Fondo de Cultura Económica.

- Colomer, T. y Munita, F. (20I3). La experiencia lectora de los alumnos de magisterio: nuevos desafíos para la formación docente. Revista lenguaje y textos, 38, 37-46.

- Contreras, E. y Prats, M. (2015). ¿La educación literaria de los futuros maestros es suficiente para ejercer de mediadores de las nuevas generaciones? Via Atlantica, 28, 29-44.

- Creswell, J.W. (2012). Qualitative inquiry and research design: choosing among five traditions. Thousand Oaks: Sage.

- Delbrayelle, A. y Duszynski, M. (2007). De la difficulté à se construire comme un sujet lecteur didactique quand on est professeur des écoles stagiaire. En M. Lebrun, A. Rouxel, \& C. Vargas (Dir.), La littérature et l'école. Enjeux, résistances, perspectives (pp. IOI-III). Aixen-Provence: Publications de l'Université de Provence.

- Díaz-Plaja, A. y Prats, M. (20I3). Recordar, aprender, practicar: qué sabe y qué debe saber un futuro maestro. Revista lenguaje y textos, 38, 19-28.

- Dueñas, J., Tabernero, R., Calvo, V. y Consejo, E. (2OI4). La lectura literaria ante nuevos retos: canon y mediación en la trayectoria de los futuros profesores. Ocnos, II, 2I-43.

- Feuerstein, R. y Feuerstein, S. (I99I). Mediated learning experience: a theoretical review. En Feuerstein, R.; Klein, P. y Tennenbaum, A. (ed.). Mediated learning experience (MLE): theoretical, psychosocial and learning implications (pp. 3-5I). London: Freund Publishing House. 
- Granado, C. y Puig, M. (20I5). La identidad lectora de los maestros en formación como componente de su identidad docente. Un estudio de sus autobiografías como lectores. $O c$ nos, $\mathrm{I} 3,43-63$.

https://doi.org/IO.I8239/ocnos_2OI5.I3.O3

- Larrañaga, E. y Yubero, S. (2005). El hábito lector como actitud. El origen de "falsos lectores". Ocnos, I, 43-6o.

https://doi.org/IO.I8239/ocnos_2OO5.OI.O4

- Manresa, M. (20I3). L'univers lector adolescent. Barcelona: Rosa Sensat.

- Mar, R.A., Oatley, K., Hirsh, J., dela Paz, J., \& Peterson, J.B. (2006). Bookworms versus Nerds: Exposure to Fiction versus Non-Fiction, Divergent Associations with Social Ability, and the Simulation of Fictional Social Worlds. Journal of Research in Personality, $40(5), 694-7 \mathrm{I} 2$.

https://doi.org/ı.ІІ0I6/j.jrp.2005.08.002

- Mendoza, A. (2002) Las funciones del profesor de literatura. Bases para la innovación. En B. Muñoz (et al.) Aspectos didácticos de Lengua y Literatura, I2, (pp.Io9-I40) Zaragoza: Publicaciones ICE Universidad de Zaragoza.

- Mendoza, A. (2004). La educación literaria: bases para la formación de la competencia lecto-literaria. Málaga: Aljibe.

- Munita, F. (2OI3). Jo, lector. Els relats de vida lectora en la construcción del subjecte didàctic. Articles De Didáctica de la Llengua i de la Literatura, 6I , I7-25.

- Munita, F. (2014). El mediador escolar de lectura literaria. Un estudio del espacio de encuentro entre prácticas didácticas, sistemas de creencias y prácticas personales de lectura. (Tesis doctoral). Obtenido desde Tesis Doctorals en Xarxa en 2O2O:

https://www.tdx.cat/handle/Io8o3/3I345 ${ }^{\text {I }}$ page= $=$ I

- Munita, F. (2OI8). El sujeto lector didáctico: “lectores que enseñan y profesores que leen”. Alabe, $\mathrm{I}$, , I- I9. http://doi.org/IO.I5645/Alabe2OI8.I7.2

- Munita, F. \& Manresa, M. (2OI2). La mediación en la discusión literaria. En T. Colomer y M. Fittipaldi (coords.). La literatura que acoge: Inmigración y lectura de álbumes (pp. 19I43). Barcelona: Banco del libro-Gretel.

- Munita, F. \& Margallo, A. M. (20I9). La didáctica de la literatura. Configuración de la disciplina y tendencias de investigación en el ámbito hispanohablante. Perfiles educativos, XLI(I64), I54-I7\%. 
- Naji, J.; Subramaniam, G.; White, G. (20I9). New approaches to literature for language learning. Cham: Palgrave Macmillan. https://doi.org/IO.IOO7/978-3-O3O-I5256-7

- Navarro Asensio, E. (coord) (20I7). Fundamentos de la investigación y la innovación educativa. Logroño: Unir Editorial.

- Palou, J. y Fons, M. (20IO, junio, IO y II). Relats de vida lingüística en els processos de formació dels docents. En I Jornadas de Historias de Vida en Educación Cuestiones epistemológicas, metodológicas, éticas y de formación, Barcelona, España.

- QSR International Pty Ltd, C. (20I6). NVivo Qualitative Data Analysis Software.

- Rain, M. y Mar, R.A. (20I4). Measuring Reading Behavior: Examining the Predictive Validity of Print-Exposure Checklists. Empirical Studies of the Arts, 32(I), 93-Io8. https://doi.org/ıo.2190/em.32.If

- Sandín, M.P. (2003). Investigación cualitativa en educación: fundamentos y tradiciones. McGraw-Hill: Madrid.

- Sanjuán Álvarez, M. (20I6). Los factores emocionales en el aprendizaje literario. En J.L. Soler, L. Aparicio, O. Díaz, E. Escolano y A. Rodríguez (coords.), Inteligencia emocional y bienestar II: reflexiones, experiencias profesionales e investigaciones (pp.I56-I7I). Zaragoza: Ediciones Universidad San Jorge.

- Trotman, J. y Kerr, T (200I). Making the Personal Professional: Pre-service teacher education and personal histories. Teachers and Teaching. Theory and Practice. 7, (2), I57-I7I.

- Vygotski, L. (2009/1960). Eldesarrollo de los procesos psicológicos superiores. Barcelona: Crítica. 